\title{
Cardiac-specific ablation of ARNT leads to lipotoxicity and cardiomyopathy
}

\author{
Rongxue Wu, ${ }^{1}$ Hsiang-Chun Chang, ${ }^{1}$ Arineh Khechaduri, ${ }^{1}$ Kusum Chawla, ${ }^{1}$ Minh Tran, ${ }^{1}$ Xiaomeng Chai, ${ }^{1}$ Cory Wagg, ${ }^{2}$ \\ Mohsen Ghanefar, ${ }^{1}$ Xinghang Jiang, ${ }^{1}$ Marina Bayeva, ${ }^{1}$ Frank Gonzalez, ${ }^{3}$ Gary Lopaschuk, ${ }^{2}$ and Hossein Ardehali ${ }^{1}$
}

'Division of Cardiology, Department of Medicine, Northwestern University School of Medicine, Chicago, Illinois, USA. ²ardiovascular Research Centre, Mazankowski Alberta Heart Institute, University of

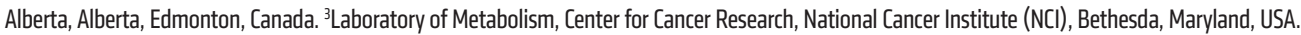

\begin{abstract}
Patients with type 2 diabetes often present with cardiovascular complications; however, it is not clear how diabetes promotes cardiac dysfunction. In murine models, deletion of the gene encoding aryl hydrocarbon nuclear translocator (ARNT, also known as HIF1 $\beta$ ) in the liver or pancreas leads to a diabetic phenotype; however, the role of ARNT in cardiac metabolism is unknown. Here, we determined that cardiac-specific deletion of Arnt in adult mice results in rapid development of cardiomyopathy (CM) that is characterized by accumulation of lipid droplets. Compared with hearts from ARNT-expressing mice, ex vivo analysis of ARNT-deficient hearts revealed a 2-fold increase in fatty acid (FA) oxidation as well as a substantial increase in the expression of PPAR $\alpha$ and its target genes. Furthermore, deletion of both Arnt and Ppara preserved cardiac function, improved survival, and completely reversed the FA accumulation phenotype, indicating that PPAR $\alpha$ mediates the detrimental effects of Arnt deletion in the heart. Finally, we determined that ARNT directly regulates Ppara expression by binding to its promoter and forming a complex with HIF2 $\alpha$. Together, these findings suggest that ARNT is a critical regulator of myocardial FA metabolism and that its deletion leads to CM and an increase in triglyceride accumulation through PPARa.
\end{abstract}

\section{Introduction}

Diabetes mellitus is a common disorder, with approximately 300 million people worldwide believed to be affected (1). Type 2 diabetes is associated with cardiovascular complications, including an increased incidence of heart failure after myocardial infarction or even in the absence of coronary artery disease (2). The mechanism linking diabetes to heart dysfunction is not entirely clear, and several hypotheses have been proposed, including microvascular disease, glucose toxicity, mitochondrial dysfunction, and lipid toxicity. The latter mechanism is supported by studies showing that lipid accumulation and excessive fatty acid oxidation (FAO) contribute to cardiomyocyte dysfunction $(3,4)$. Furthermore, the hearts of diabetic animals and patients cannot fully utilize glucose, resulting in increased fatty acid uptake (FAU) and FAO and lipid deposition (3). The cardiomyopathy (CM) that is associated with these features is termed lipotoxic CM.

Aryl hydrocarbon nuclear translocator (ARNT), also known as hypoxia-inducible factor $1 \beta$ (HIF1 $\beta$ ), belongs to the basic helix -loop-helix (bHLH) family of transcription factors and heterodimerizes with several other bHLH family members, including HIF1 $\alpha$, HIF2 $\alpha$, and the aryl hydrocarbon receptor (AHR) (5-7). In response to hypoxia, ARNT binds to HIF1/2 $\alpha$ and interacts with hypoxia response elements (HREs) to activate the transcription of genes needed for adaptation to oxygen deprivation and for cell survival under hypoxia. ARNT also dimerizes with AHR, and this heterodimer plays a role in the development and detoxification of

Conflict of interest: Hossein Ardehali receives speaking honoraria from Merck and consulting honoraria from Cubist Pharmaceuticals and Takeda.

Submitted: April 23, 2014; Accepted: September 8, 2014.

Reference information: / Clin Invest. 2014;124(11):4795-4806. doi:10.1172/JCI76737. a wide array of xenobiotics (8). The homozygous deletion of Arnt in mice leads to embryonic lethality due to defects in angiogenesis and an impaired response to glucose deprivation and hypoxia (9). Interestingly, reduced ARNT expression has been observed in the pancreas and liver of humans with type 2 diabetes, and Arnt deletion in the pancreas and liver of mice leads to a condition that mimics type 2 diabetes $(10,11)$. These studies suggest that ARNT deficiency plays an important role in the altered metabolic function associated with diabetes, however, the mechanism for this phenomenon is not well characterized. Furthermore, the role of ARNT in the metabolism of other insulin-responsive tissues, such as the heart, has not been studied.

Peroxisome proliferator-activated receptors (PPARs) are ligand-activated transcription factors that belong to the nuclear receptor superfamily and play an important role in the regulation of cellular metabolism (12). PPAR $\alpha$ controls the expression of a wide range of proteins involved in both the transport and $\beta$ oxidation of free FAs in liver, kidney, heart, muscle, and adipose tissue $(13,14)$ and is activated by FA moieties and fibrates $(15)$. In the vasculature, PPAR $\alpha$ exerts antiinflammatory effects in addition to having a positive influence on vessel reactivity and remodeling and macrophage lipid handling within the plaque (16-18). PPAR $\alpha$ is also known to play a role in the development of hypertension and the cardiac response to ischemia-reperfusion and pressure overload (12), and the levels of PPAR $\alpha$ and its target genes are elevated in the hearts of mouse models of diabetes (19). Ppara ${ }^{-/}$mice are viable and have normal cardiac function at baseline, however, they exhibit lipid accumulation in their tissues in response to inhibition of mitochondrial FA import (20). Transgenic (Tg) mice that overexpress PPAR $\alpha$ in their hearts display phenotypic features similar to those observed in diabetic hearts (19), 
including increased myocardial FAU and FAO, reduced glucose utilization, and CM (21). They also exhibit FA deposits throughout their heart, which is believed to be due to an imbalance between FAU and FAO, both of which are increased to different degrees. Furthermore, the CM seen in PPAR $\alpha$ Tg mice is exacerbated by a high-fat diet, suggesting that FA overload contributes to the lipotoxicity and cardiac dysfunction (22). However, it is not known whether an increase in PPAR $\alpha$ through non-Tg models would still result in the same phenotype.

Here, we demonstrate that deletion of Arnt in the heart leads to $\mathrm{CM}$ and lipid accumulation. Our mechanistic studies determined that PPAR $\alpha$ mediates the deleterious effects and metabolic changes of Arnt deletion in the heart, as deletion of PPAR $\alpha$ reverses the phenotype associated with Arnt knockout. Furthermore, we demonstrate that ARNT regulates PPAR $\alpha$ at the transcriptional level by binding to its promoter and by forming a complex with HIF $2 \alpha$. We believe that these findings provide the first evidence for a functional link between the HIF/ARNT pathway and PPAR $\alpha$ in the heart and demonstrate that deletion of Arnt leads to CM and cardiac lipotoxicity through PPAR $\alpha$ activation.

\section{Results}

Cardiac deletion of Arnt leads to CM. ARNT levels are reduced in the liver and pancreas in diabetes, and its deletion in those tissues leads to a diabetic phenotype $(10,11)$. However, the function of ARNT in the heart is not known. We first assessed the levels of ARNT in hearts from diabetic mice. The levels of ARNT were significantly decreased in $\mathrm{db} / \mathrm{db}$ mice at 32 weeks of age compared with those detected in age-matched WT animals (Figure 1A). To study the role of ARNT in the heart, we generated tamoxifeninducible cardiac-specific ARNT-knockout mice (csArnt $\left.{ }^{-/}\right)$by crossing mice with loxP sequences flanking exon 8 of $\operatorname{Arnt}\left(\right.$ Arnt $\left.t^{f / f}\right)$ (23) with mutated estrogen receptor (MER)-Cre-MER (MCM) Tg mice under the $\alpha$-myosin heavy chain (Myh6) promoter (24) (Figure 1, B and C, and Supplemental Figure 1A; supplemental material available online with this article; doi:10.1172/ JCI76737DS1). Intraperitoneal injection of tamoxifen in these mice resulted in transient but severe CM independent of Arnt deletion, consistent with previous work (25). Thus, we adopted a 2-week protocol of oral tamoxifen administration (Supplemental Figure 1B), which did not result in any changes in body weight (Supplemental Figure 1C). Deletion of Arnt in the heart was confirmed at the genomic DNA (Figure 1D), mRNA (Figure 1E and Supplemental Figure 2A), and protein levels (Figure 1F). We found that deletion of Arnt was specific to the heart, as its mRNA and protein levels in other tissues were comparable to those in the control mice (Supplemental Figure 2, B and C).

We then assessed the effects of Arnt deletion on cardiac function. A dilated left ventricle in $\mathrm{csArnt}^{-/-}$mice was observed at 4 weeks after tamoxifen treatment, while all control group mice displayed normal cardiac size and function (Figure 2A and Supplemental Figure 3, A-E). For subsequent studies, we used Arnt $t^{A / A l}$ mice treated with tamoxifen as controls. Echocardiographic analysis of the hearts of csArnt ${ }^{-/}$mice revealed decreased fractional shortening (FS) and ejection fraction (EF) and increased left ventricular end-diastolic internal dimensions (LVID-d) as early as 2 weeks after tamoxifen treatment, with the reduction in FS and EF persisting at least up to 4 weeks (Figure 2, B-D). cs Arnt $t^{-/}$mice also displayed a reduction in the early to late ventricular filling velocities (E/A) ratio that was measured across the mitral valve (Figure $2 \mathrm{E}$ ). Invasive hemodynamic measurements revealed increased end-diastolic pressure (EDP), while the maximum and minimum rates of pressure change in the left ventricle $(+\mathrm{dP} / \mathrm{dt}$ and $-\mathrm{dP} / \mathrm{dt})$ were significantly reduced with Arnt deletion (Figure 2, F and G), suggesting a combination of systolic and diastolic dysfunction in the hearts of $\mathrm{csArnt}^{-/}$mice. Consistent with cardiac dysfunction, $\mathrm{csArnt}^{-/-}$mice also displayed higher heart and lung weights normalized to tibia length (Supplemental Figure 4, A-C). Finally, the levels of heart failure markers atrial natriuretic protein (ANP) and brain natriuretic protein (BNP) were significantly higher in $\mathrm{csArnt}^{-1-}$ mice than in controls (Supplemental Figure 5, A and B). These results suggest that Arnt deletion in the heart leads to the development of spontaneous dilated CM.

Arnt deletion in the heart leads to excess lipid accumulation. We then studied the mechanism for the CM associated with Arnt deletion. H\&E staining showed myofiber architectural distortion and markedly increased interstitial fibrosis in csArnt ${ }^{-/-}$hearts 4 weeks after tamoxifen administration (Figure 3A). We also noted evidence of enhanced apoptosis in $\mathrm{csArnt}^{-/-}$hearts compared with that seen in control hearts, as assessed by TUNEL staining of ventricular tissue (Figure 3B). Electron microscopic analysis revealed the presence of large vacuoles scattered throughout the heart tissue of $\mathrm{csArnt}^{-/-}$mice (Figure 3C), bearing structural similarity to lipid vacuoles (26). These results suggest that Arnt deletion in the heart may lead to lipid accumulation; thus, we performed further analysis of the lipid levels in csArnt $t^{-/-}$hearts 4 weeks after initiation of tamoxifen treatment. Oil red $\mathrm{O}$ staining revealed enhanced dye accumulation in csArnt ${ }^{-/}$hearts (Figure 3, D and $\mathrm{E})$, confirming increased lipid content. Moreover, we found that triacylglycerol (TAG) levels were higher in the hearts of csArnt $t^{-/}$ mice (Figure $3 \mathrm{~F}$ ), while serum TAG levels were similar compared with those in control mice (Supplemental Figure 6A). Consistent with these results, we also found an increase in TAG levels in isolated neonatal rat cardiomyocytes (NRCMs) in response to ARNT knockdown with siRNA (Supplemental Figure 6, B and C). Taken together, these results show that Arnt deletion causes significant lipid accumulation in the heart.

Arnt deletion in the heart leads to an increase in FA metabolism. We next assessed the effects of Arnt deletion on cardiac FA and glucose metabolism in isolated working hearts using radioisotope-labeled substrates. Deletion of Arnt in the heart resulted in an approximately 2-fold increase in FAO (as assessed by oxidation of ${ }^{3} \mathrm{H}$-palmitate) (Figure $4 \mathrm{~A}$ ). $\operatorname{cs} A r n t^{-/-}$mice also displayed a decrease in glucose oxidation (Supplemental Figure 7A) and a significant reduction in genes involved in glucose metabolism (Supplemental Figure 7B). To confirm that the observed changes in glucose and FA metabolism in the csArnt $t^{-/-}$hearts were specific to the absence of cardiac ARNT and not secondary to the CM associated with Arnt deletion, we performed in vitro studies in NRCMs. Consistent with the in vivo data, ARNT knockdown in NRCMs led to increased FA uptake (FAU) (Figure 4B) and FAO (Figure 4C). Knockdown of ARNT in NRCMs also resulted in a significant reduction in glucose uptake and the extracellular acidification rate (ECAR) (Supplemental Figure 7, C and D). These results suggest 

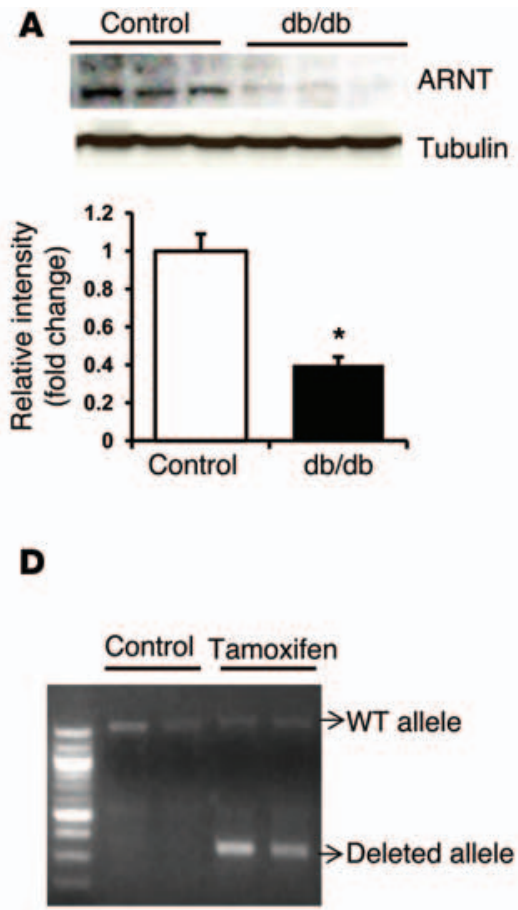

B

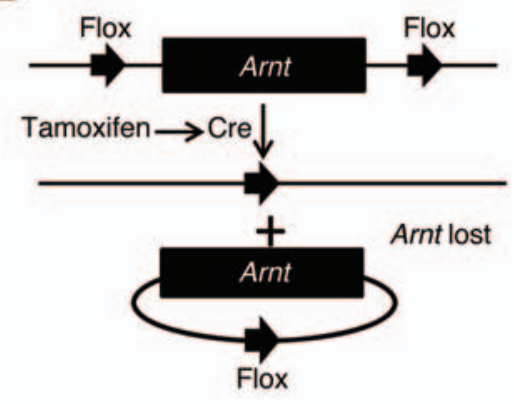

E

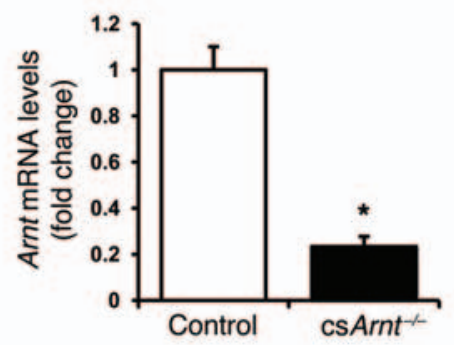

C
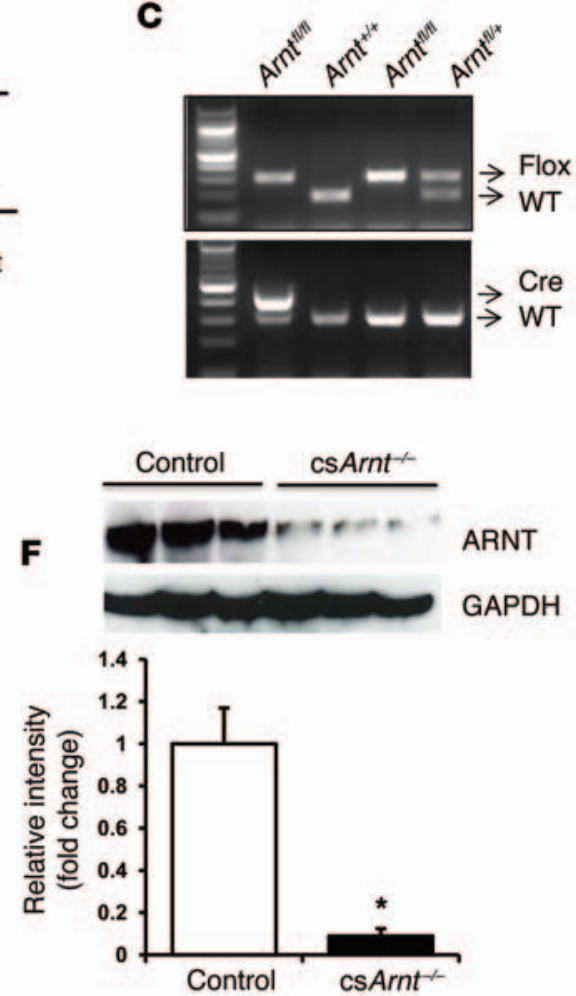

Figure 1. Generation of cardiac-specific knockout of ARNT. (A) Western blot of ARNT in the hearts of 32-week-old db/db and age-matched control mice ( $n=7$ independent experiments for the bar graph). (B) Schematic diagram of Arnt deletion in the heart. Mice with loxP sequences flanking exon 8 of Arnt were crossed with Mcm Tg mice under the Myh6 promoter. (C) PCR analysis of genomic DNA of Arnt homozygous and heterozygous floxed mice with or without $C r e$ and in the absence of tamoxifen. (D) PCR analysis of Arnt $t^{f / f l}$ mice after crossing with Cre and tamoxifen treatment. DNA samples from 2 normal chow-fed and 2 tamoxifen chow-fed Cre Arnt $t^{f / f l}$ mice are shown. (E) qRT-PCR results of Arnt transcript levels in control and csArnt ${ }^{-/-}$ hearts ( $n=6$ independent experiments). (F) Western blot analyses of ARNT in control and csArnt ${ }^{-/-}$hearts $(n=6$ independent experiments). A summary bar graph is shown below the blot. Data are presented as the mean $\pm \mathrm{SEM}$. ${ }^{*} P<0.01$.

that Arnt deletion in the heart results in an increase in FA metabolism and a decrease in glucose utilization.

The PPAR $\alpha$ pathway is activated in the hearts of $\mathrm{csArnt}^{-/}$mice. We next studied the mechanism for increased lipid accumulation in the hearts of $\operatorname{csArnt^{--}}$ mice. PPARs are known to regulate FA metabolism (12), thus, we examined the effects of Arnt deletion on the expression of PPAR proteins. Although Arnt deletion in the heart did not alter the mRNA levels of Pparg or Pparb/d, it did result in a significant increase in Ppara mRNA (Figure 4D) and protein (Figure 4E) levels. Similarly, knockdown of ARNT with siRNA in NRCMs resulted in a significant increase in PPAR $\alpha$ levels (Supplemental Figure 8, A and B). In addition, PPAR $\alpha$ target genes involved in mitochondrial $\beta$ oxidation, such as carnitine palmitoyltransferase 1 (Cpt1) and fatty acyl-CoA synthetase (Acs), were significantly increased in $\mathrm{csArnt}^{-/-}$hearts. Diacylglycerol acyltransferase (Dgat), the key enzyme involved in the esterification of FA to TAG, was also significantly elevated in the hearts of $\mathrm{csArnt}^{-/-}$mice (Figure 4F).

These results suggest that ARNT inhibits PPAR $\alpha$ expression. The HIF pathway generally causes activation of genes; however, recent studies suggest that HIF may also inhibit promoter activity in certain genes (27-29). To assess whether ARNT regulates PPAR $\alpha$ at the transcriptional level, we first transfected a plasmid construct of the human Ppara promoter luciferase reporter (which has 5
HIF binding sites) (Figure 5A) or a control plasmid into HEK293 cells, along with control or ARNT siRNA. ARNT siRNA resulted in effective knockdown in HEK293 cells (Supplemental Figure 9A) and led to a significant increase in luciferase activity (Figure 5B), suggesting that ARNT likely regulates PPAR $\alpha$ at the mRNA level. We then assessed which of the HREs is responsible for the inhibitory effects of ARNT on the Ppara promoter by making truncated forms of the promoter to sequentially remove HREs (Figure 5A). A truncated construct with removal of the second HRE upstream of the initiation site abolished the inhibitory effect of ARNT on the Ppara promoter, suggesting that this site is likely involved in the regulation of the gene (Figure $5 \mathrm{~B}$ ). To confirm this observation, we performed ChIP analysis on this HRE. Negative and positive controls for the ChIP are shown in Supplemental Figures 9, B and C, and ChIP analysis of the second HRE upstream of the Ppara initiation site confirmed that ARNT binds to this sequence (Figure 5C).

To determine which ARNT partner mediates its effects on PPAR $\alpha$ expression, we then downregulated HIF1 $\alpha$, HIF $2 \alpha$, and AHR in NRCMs and assessed PPAR $\alpha$ levels. A reduction in HIF1 $\alpha$ or AHR did not alter the levels of PPAR $\alpha$ significantly, while HIF $2 \alpha$ knockdown, similar to the effects of ARNT reduction, resulted in an increase in PPAR $\alpha$ levels (Supplemental Figure 10, A-D, and Figure 5D). These results suggest that the effects of Arnt deletion on PPAR $\alpha$ are, at least partially, through its association with 
A

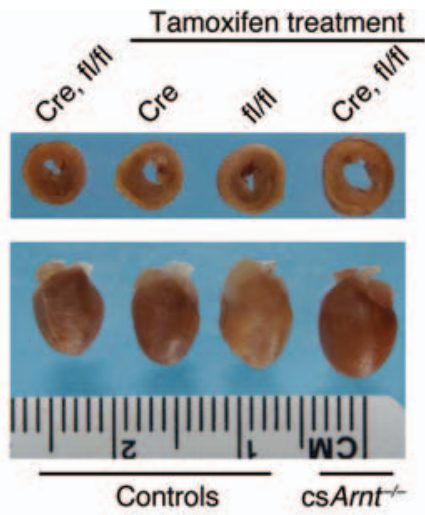

B

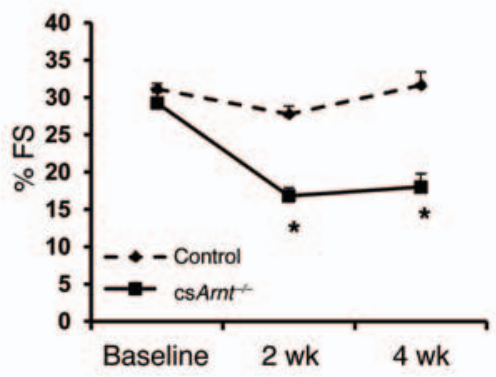

C

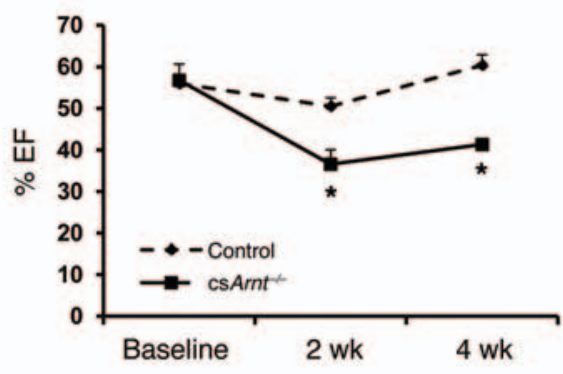

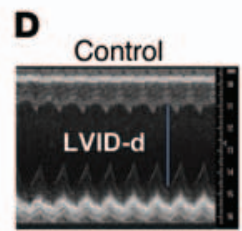

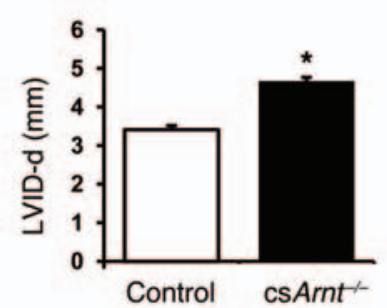

E
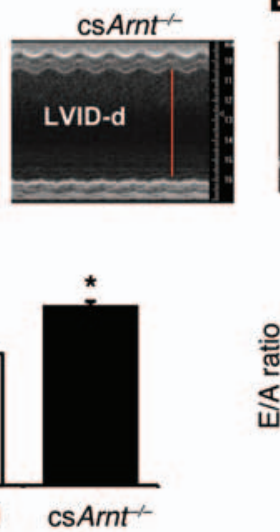
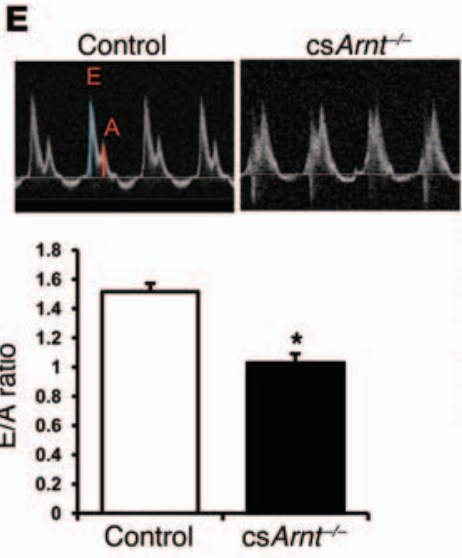

$\mathbf{F}$

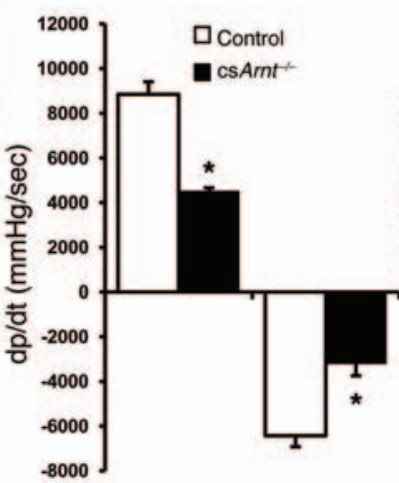

G

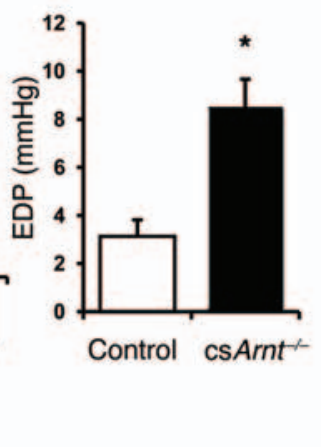

Figure 2. Deletion of Arnt in the heart leads to CM. (A) Gross images of hearts from 3 sets of controls (Cre, Arnt ${ }^{f / f l}$ on a normal chow diet, Cre Tg mice on a tamoxifen-treated chow diet, and Arnt $f^{f / f l}$ mice on a tamoxifen chow diet) and csArnt ${ }^{-/-}$mice. (B-E) Echocardiographic data on control (Arnt ${ }^{f / f l}$ mice treated with tamoxifen) and csArnt ${ }^{-/-}$mice ( $n=12$ independent experiments). FS (B), EF (C), LVID-d (D), and E/A ratio (E), as assessed by echocardiography ( $n=12$ independent experiments). FS and EF were measured 2 and 4 weeks after completion of tamoxifen treatment, while LVID-d and the E/A ratio were assessed at 4 weeks. LVID-d and the E/A ratio are summarized in bar graphs below the figures. Positive and negative dp/dt (F) and EDP (G) using invasive hemodynamic measurements in control and csArnt ${ }^{-/-}$mice ( $n=6$ independent experiments). Data are presented as the mean \pm SEM. ${ }^{*} P<0.01$.

HIF $2 \alpha$ and through the binding of this complex to the second HRE upstream of the Ppara initiation site. To confirm these results, we then performed sequential ChIP studies using HIF $2 \alpha$ and ARNT antibodies on the second HRE sequence. As shown in Figure 5E, ARNT and HIF $2 \alpha$ bound to this HRE sequence as a complex. Finally, we either deleted or mutated the second HRE in the fulllength Ppara promoter attached to luciferase and showed that both of these changes resulted in a reversal in the increase in luciferase activity with ARNT kno(Figure 5, F and G). These results provide evidence that the second HRE mediates the effects of ARNT/HIF $2 \alpha$ on the Ppara promoter.

We next assessed whether the effects of ARNT knockdown on the Ppara promoter occurs through changes in the activity of histone deacetylases (HDACs). We showed that treatment of cells with the HDAC inhibitor trichostatin A (TSA) did not alter Ppara mRNA levels in the presence or absence of ARNT knockdown (Supplemental Figure 11). Furthermore, we found that HDAC1-4 levels were not changed with ARNT knockdown (Supplemental Figure 12, A and B), and ChIP studies showed that the levels of acetylated histones $\mathrm{H} 3$ and $\mathrm{H} 4$ were not increased on the Ppara promoter after ARNT knockdown (Supplemental Figure 12, C-E). Thus, the effects of ARNT on the Ppara promoter are independent of HDACs and histone deacetylation.
Deletion of Ppara prevents cardiac metabolic derangements associated with Arnt deletion. We next assessed whether PPAR $\alpha$ is increased in the hearts of diabetic mice and showed that its mRNA levels were higher in hearts from $\mathrm{db} / \mathrm{db}$ mice (Supplemental Figure 13). These findings are consistent with studies by Finck et al., who demonstrated that PPAR $\alpha$ mRNA and protein levels are increased in streptozotocin-treated hearts and that PPAR $\alpha$ target genes (but not its own mRNA levels) are increased in $\mathrm{db} / \mathrm{db}$ hearts (19). To better assess the role of PPAR $\alpha$ in the metabolic effects of ARNT in the heart, we knocked down ARNT or PPAR $\alpha$ individually or together in NRCMs, then measured the genes involved in FA metabolism. Deletion of Ppara either completely or partially reversed the effects of Arnt deletion on genes involved in FA metabolism (Figure 6A). Furthermore, we found that the increase in FAO (as assessed by oxygen consumption in the presence of palmitate) with ARNT knockdown was completely reversed with Ppara deletion (Figure 6B). Also, csArnt $t^{-/}$hearts displayed higher levels of UCP2 and UCP3 (Supplemental Figure 14), suggesting that the hearts of these mice have more uncoupling and inefficient fuel use.

To confirm that myocardial lipid accumulation and FAO associated with Arnt deletion in the heart are PPAR $\alpha$ dependent, we generated csArnt ${ }^{-/-}$and $\mathrm{Ppara}^{-/-}$double-knockout mice by crossing Myh6-MCM Arnt $t^{A / f}$ mice with $\mathrm{Ppara}^{-/-}$mice, followed 
A

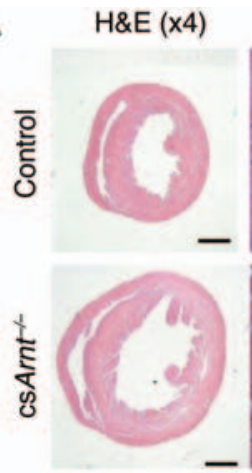

$H \& E(x 200)$

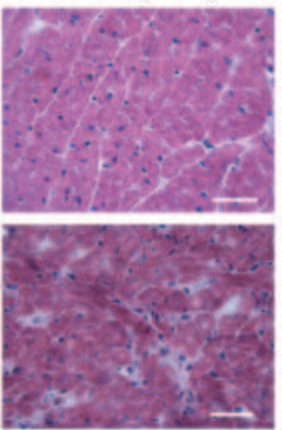

MT (x200)

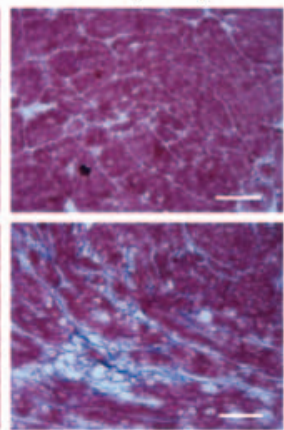

B
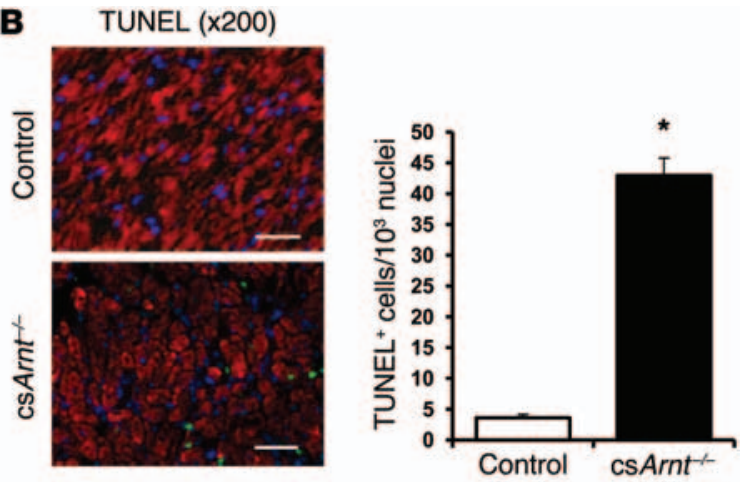

E

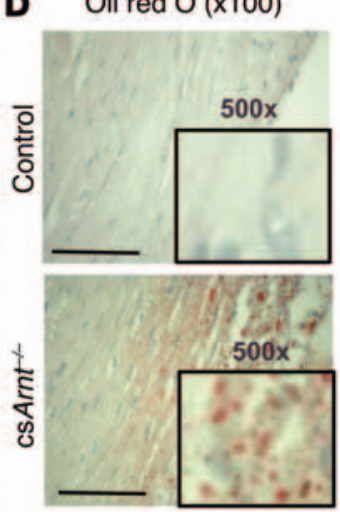

$(x 9,300)$

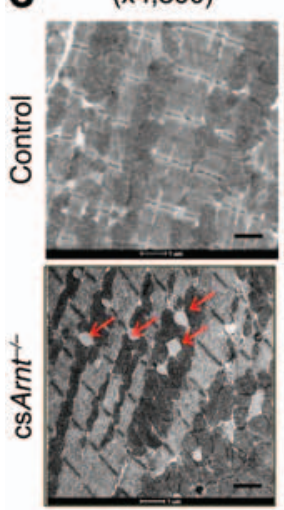

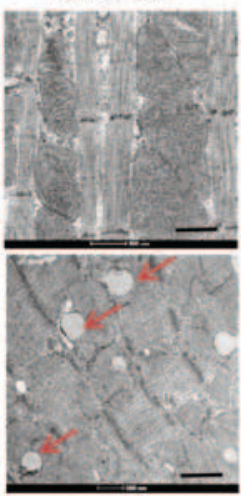

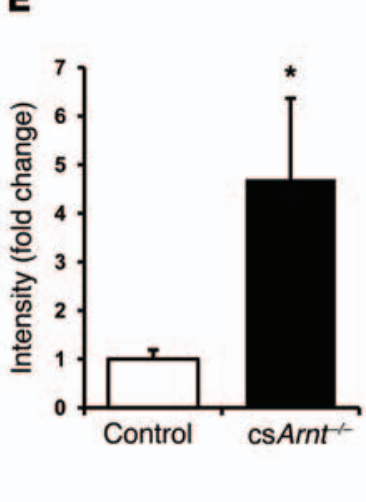

$\mathbf{F}$

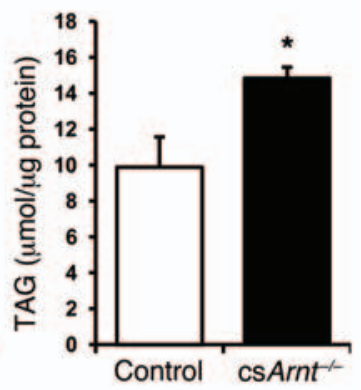

Figure 3. csArnt ${ }^{-/-}$hearts display increased lipid accumulation. (A) Representative cardiac histological sections of control (Arnt $t^{f / f f}$ littermates) and csArnt ${ }^{-/-}$mice 4 weeks after initiation of tamoxifen treatment. MT, Masson's trichrome. Scale bars: $1 \mathrm{~mm}(\mathrm{H} \& \mathrm{E}-\mathrm{stained} \times 4$ images) and $100 \mu \mathrm{m}(\mathrm{H} \& \mathrm{E}-$ stained $\times 200$ and MT-stained images). Experiments were repeated 3 times. (B) TUNEL staining of hearts from control and csArnt ${ }^{-1-}$ mice. TUNEL ${ }^{+}$cells were normalized to the total number of nuclei and are represented as a bar graph next to the images $(n=6$ independent experiments). Scale bars: $500 \mu \mathrm{m}$. (C) Electron microscopic images of control and csArnt ${ }^{-1-}$ hearts indicated the presence of fat droplets. Red arrows point to lipid vacuoles in the $\mathrm{cs} \mathrm{Arnt}^{-1-}$ hearts. Scale bars: $1 \mu \mathrm{m}$ ( $\times 4,800$ images) and $500 \mathrm{~nm}\left(\times 9,300\right.$ images). (D) Oil red 0 staining of control and csArnt ${ }^{-/}$hearts. Scale bars: $500 \mu \mathrm{m}$ (original magnification, $\times 100$ and $\times 500$ [insets]). Experiments were repeated 3 times. (E) Summary bar graph of images in $\mathbf{D}(n=3$ independent experiments). (F) TAG content in the heart of control and csArnt ${ }^{-/-}$mice $\left(n=6\right.$ independent experiments). Data are presented as the mean \pm SEM. ${ }^{*} P<0.01$.

by tamoxifen treatment (Supplemental Figure 15A). The genotype of the double-knockout mice was confirmed by PCR, and deletion of the genes was confirmed by measuring the levels of both proteins in the hearts of double-knockout mice with double immunofluorescence (Supplemental Figure 15, B and C). We then measured substrate metabolism in the hearts of these mice using a working heart model and ${ }^{3} \mathrm{H}$-labeled palmitate. As expected, FAO was significantly higher in $\mathrm{csArnt}^{-/-}$hearts compared with that in control hearts. Deletion of PPAR $\alpha$ in csArnt ${ }^{-/-}$ hearts reversed the increase in myocardial TAG content (Figure 6C), FAO (Figure 6D), and gene targets of PPAR $\alpha$, including Cpt1 and Acs (Figure 6E). Moreover, csArnt ${ }^{-/} \mathrm{Ppara}^{-/}$doubleknockout mice displayed significantly lower lipid accumulation compared with csArnt ${ }^{-/}$mice, as assessed by oil red O staining (Figure 6F). Since myocardial cell death is a consequence of lipotoxicity in the heart, we assessed myocardial cell death and interstitial fibrosis. Histologic examination of left ventricular tissue revealed that deletion of Ppara normalized myocardial cytoarchitecture and reversed cell death, as determined by TUNEL staining (Figure 6, F and G). Collectively, these results provide in vitro and in vivo data showing that PPAR $\alpha$ mediates the lipid accumulation and increase in FAO associated with ARNT knockdown.

Cardiac function is restored with Ppara deletion in $\mathrm{csArnt}^{-1-}$ mice. Finally, we sought to determine whether the CM observed in $\mathrm{csArnt}^{-1-}$ mice is due to PPAR $\alpha$ activation. We assessed cardiac function by echocardiography in the $\mathrm{csArnt}^{-/-}$and $\operatorname{csArnt^{-/-}}$ Ppara $^{-/-}$double-knockout mice 1, 2, and 4 weeks after tamoxifen administration. While csArnt $t^{-/}$mice displayed CM 4 weeks after tamoxifen treatment, csArnt ${ }^{-/} \mathrm{Ppara}^{-/}$double-knockout mice showed a complete reversal of $\mathrm{CM}$, as determined by cardiac output, EF, and FS (Figure 7, A-D, and Supplemental Table 1). Moreover, the molecular markers of heart failure, ANP and BNP, were also reduced upon PPAR $\alpha$ deletion (Supplemental Figure 16, A and B). Finally, Kaplan-Meier analysis showed that csArnt ${ }^{-1-}$ $\mathrm{Ppara}^{-/-}$double-knockout mice had no mortality by 2 months of age, while $\mathrm{csArnt}^{-/-}$mice had approximately $15 \%$ mortality by that time (Figure 7E). These results suggest that Arnt deletion leads to cardiac dysfunction, which is mediated through PPAR $\alpha$-mediated lipid accumulation (Figure 7F). 
A

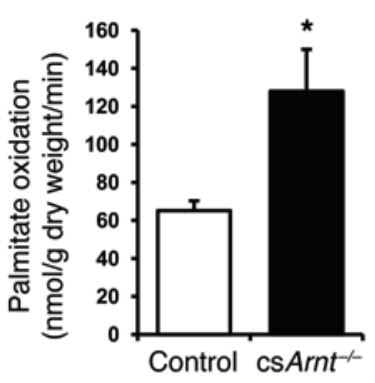

B

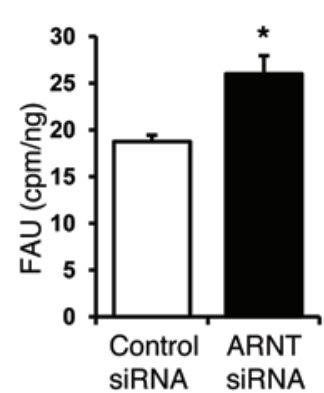

C

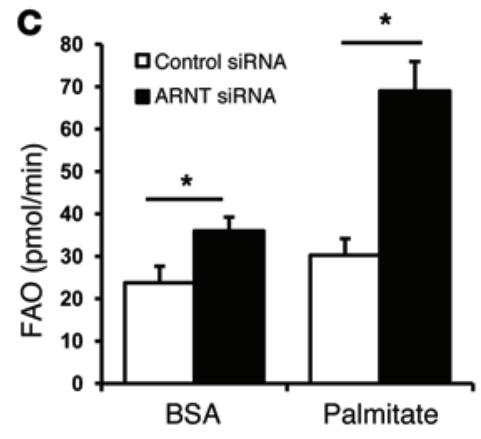

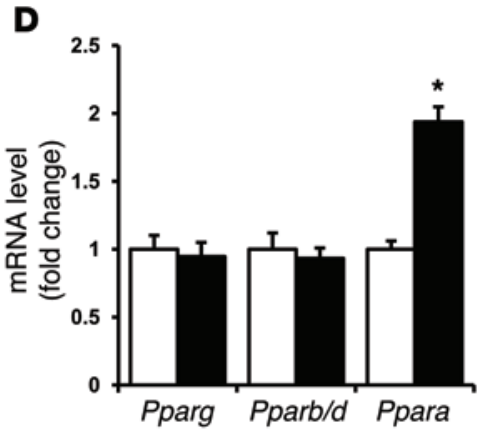

\section{E}

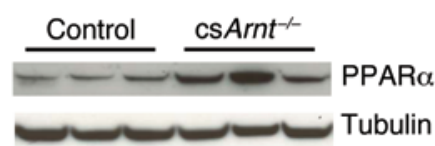

$\mathbf{F}$
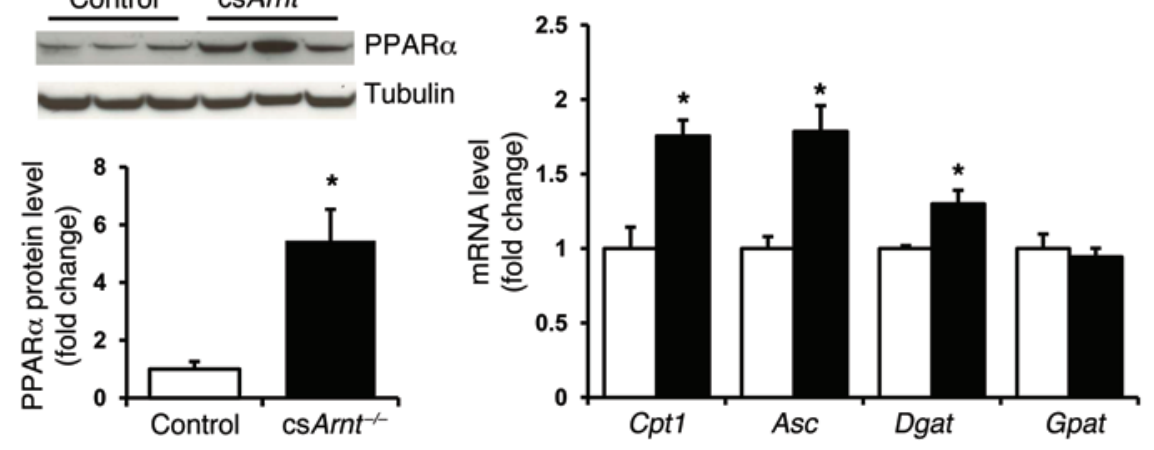

Figure 4. csArnt ${ }^{-/-}$hearts display increased FAO and PPAR $\alpha$ levels. (A) Myocardial FAO in control and csArnt ${ }^{-/-}$hearts measured in isolated working hearts using ${ }^{3} \mathrm{H}$-labeled palmitate ( $n=5-6$ independent experiments). (B) FAU in NRCMs treated with control or ARNT siRNA ( $n=6$ independent experiments). (C) FAO in control or ARNT siRNA-treated NRCMs, assessed by oxygen consumption with the addition of exogenous palmitate ( $n=5-6$ independent experiments). (D) qRT-PCR of Pparg, Pparb/d, and Ppara in NRCMs treated with control or ARNT siRNA ( $n=6$ independent experiments). (E) Western blot analysis of PPAR $\alpha$ expression in the hearts of $c s A r n t^{---}$and control mice ( $n=5$ independent experiments). (F) qRT-PCR of genes involved in FA metabolism in NRCMs treated with control or ARNT siRNA ( $n=6$ independent experiments). Data are presented as the mean \pm SEM. ${ }^{*} P<0.01$.

\section{Discussion}

Here, we showed that $\operatorname{csArnt^{-/}}$ mice display enlarged left ventricular size and reduced cardiac function, consistent with $\mathrm{CM}$. csArnt ${ }^{-1}$ hearts also exhibited lipid accumulation, as assessed by electron microscopy, oil red O staining, and TAG measurements. Using an ex vivo working heart system, we demonstrated that csArnt ${ }^{-/}$hearts have an approximately 2-fold increase in FAO and a decrease in glucose metabolism. In addition, we showed that PPAR $\alpha$ and its target genes involved in FAU and FAO are significantly increased in the csArnt ${ }^{-1}$ hearts. To confirm that the effects of ARNT downregulation on cardiac metabolism are through PPAR $\alpha$, we crossed csArnt ${ }^{--}$with $\mathrm{Ppara}^{-/-}$mice and showed that knockout of Ppara reverses the pathologic changes associated with ARNT reduction. Finally, we showed that ARNT inhibits Ppara promoter activity by forming a complex with HIF 2 . We conclude that ARNT is a critical regulator of myocardial FA metabolism and that a reduction in ARNT levels leads to cardiac dysfunction at baseline by increasing TAG accumulation through a PPAR $\alpha$-mediated pathway.

The mechanism for an increased incidence of heart dysfunction in diabetes is not totally clear, and several hypotheses have been proposed. Lipid toxicity has been suggested as a potential mechanism, an idea that is supported by studies showing that excessive FA accumulation and oxidation contribute to cardiomyocyte dysfunction. Furthermore, glucose uptake and utilization are reduced in diabetic hearts, while there is a concurrent increase in FAU and metabolism, leading to lipotoxic CM. Overexpression of PPAR $\alpha$ results in a phenotype similar to that of diabetic cardiac dysfunction, and PPAR $\alpha$ levels are increased in the hearts of diabetic mice (19), raising the possibility that PPAR $\alpha$ plays a major role in the pathogenesis of lipotoxicity associated with diabetic CM. In our studies, we also noted an increase in PPAR $\alpha$ with Arnt deletion, which was associated with lipid accumulation in the heart. It is important to point out that the extent of the PPAR $\alpha$ increase in the $\mathrm{csArnt}^{-1-}$ mice was only approximately 5- to 6-fold at the protein level (Figure 4E), which was associated with a significant reduction in cardiac function. This phenotype is not totally consistent with what was observed in PPAR $\alpha$ Tg mice, which displayed a greater than 15-fold increase in PPARa levels in the heart and a milder form of CM compared with $\mathrm{csArnt}^{-1}$ mice (19). Thus, in addition to a PPAR $\alpha$-dependent pathway, other mechanisms (independent of PPAR $\alpha$ ) may contribute to the pathogenesis of $\mathrm{CM}$ associated with Arnt deletion. Nevertheless, our findings provide evidence that even an indirect increase in the levels of PPAR $\alpha$ (as opposed to direct overexpression in $\mathrm{Tg}$ animals) contributes to lipotoxicity and CM, providing a further link between PPAR $\alpha$ and the diabetic CM phenotype.

The active HIF complex is composed of 2 subunits: ARNT and either HIF1 $\alpha$ or HIF 2 . In the ischemic heart, the HIF/ARNT complex participates in the cardiomyocyte response to low oxygen tension by upregulating glucose transporters and glycolytic enzymes (30). Global Hifla knockout results in embryonic lethal- 

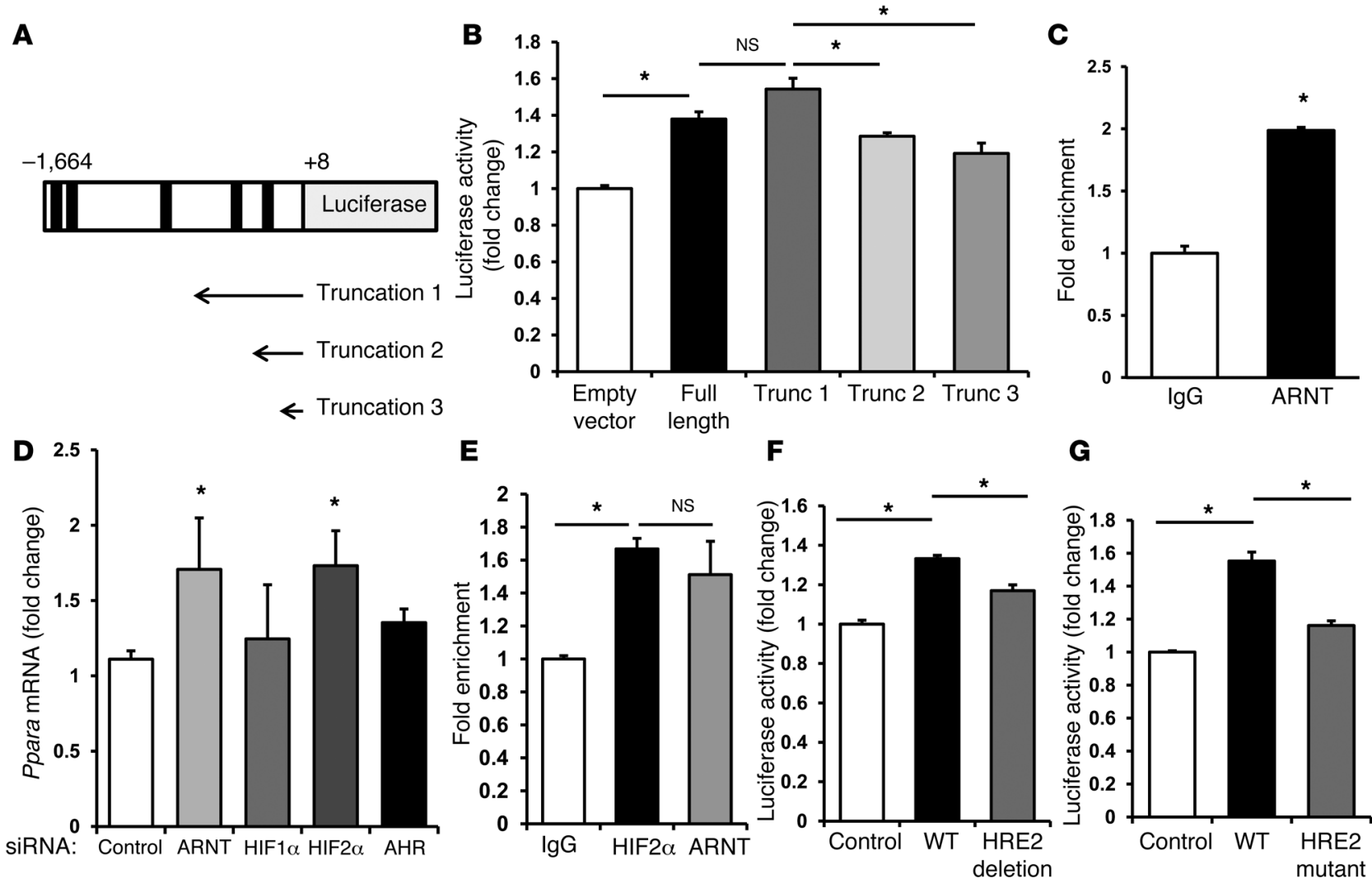

Figure 5. ARNT regulates PPAR at the transcriptional level. (A) Schematic presentation of the Ppara promoter depicting its HRE sequences. (B) Relative luciferase activity in cells treated with control or ARNT siRNA and transfected with a Ppara promoter-luciferase reporter plasmid or constructs with a truncated Ppara promoter. Renilla luciferase activity was used as an internal control and for normalization of transfection efficiency ( $n=6$ independent experiments). Data are presented as the fold change of luciferase activity in ARNT siRNA-treated cells over control siRNA-treated cells and normalized to that of the empty vector reporter. Trunc, truncation. (C) Results of ChIP on the second HRE upstream of the PPAR $\alpha$ initiation site and using ARNT antibody ( $n=3$ independent experiments). (D) Changes in Ppara mRNA levels in response to knockdown of ARNT and its partners in NRCMs ( $n=6$ independent experiments). (E) Sequential ChIP studies using HIF2 $\alpha$ and ARNT antibodies. The first ChIP was performed with HIF2 $\alpha$ antibody, and the subsequent ChIP was conducted with the indicated antibodies ( $n=3$ independent experiments). (F) Luciferase activity of WT and a construct with the deletion of the second HRE in cells treated with either control or ARNT siRNA ( $n=6$ independent experiments). (G) Luciferase activity of WT and a construct with mutations in the second HRE in cells treated with either control or ARNT siRNA ( $n=6$ independent experiments). Data in $\mathbf{F}$ and $\mathbf{G}$ are represented as fold change of luciferase activity in ARNT siRNA-treated cells over control siRNA-treated cells and normalized to that of the empty vector reporter. Data are presented as the mean \pm SEM. ${ }^{*} P<0.01$.

ity with failure of neural tube closure, defective vascularization, and increased thickness of the heart in embryos $(31,32)$. Cardiacspecific Hifla knockout leads to mild cardiac hypertrophy with reductions in contractility, vascularization, and high-energy phosphate content (33), but no evidence of lipid accumulation. However, inducible knockout of Hifla after birth in the heart does not cause cardiac dysfunction (34), suggesting that baseline HIF1 $\alpha$ function may be more important during development than in the maintenance of adult cardiac function. Mice with global Hif $2 a$ knockout display changes in different organs, including lipid accumulation in skeletal muscle, cardiac muscle, and liver (35). These mice also have cardiac hypertrophy, although cardiac function was not assessed directly (35). We showed that deletion of Arnt in the adult heart leads to spontaneous CM due to lipid accumulation, which is distinct from what is observed with HIF1 $\alpha$ deletion, but is more similar to that of Hif2a-knockout mice. In the background of Arnt deletion, it is not technically possible to reconstitute the activity of each of its partners (i.e., HIF1 $\alpha$ and $-2 \alpha$ and AHR).
However, we assessed the effects of ARNT partners in PPAR $\alpha$ regulation and demonstrated that a reduction in HIF2 $\alpha$ increases PPAR $\alpha$ levels in vitro and that ARNT forms a complex with HIF $2 \alpha$ to inhibit the Ppara promoter. Although this effect is likely independent of HDACs and histone deacetylation, it is possible that the binding of ARNT/HIF2 $\alpha$ to the Ppara promoter blocks the access of other transcription factors to their binding sites.

The link between the HIF pathway and PPARs was previously studied in the heart. Krishnan et al. showed that HIF1 $\alpha$ activates PPAR $\gamma$, which in turn, activates FAU, glucose-to-lipid conversion via the glycerol-3-phosphate pathway, apoptosis, and contractile dysfunction (36). They also demonstrated that HIF1 $\alpha$ activates PPAR $\gamma$ by binding to its promoter and that deletion of Hifa attenuates stress-induced cardiac hypertrophy. These studies focused on HIF1a, which is only one of the partners of ARNT and which was shown in our studies not to play a role in the CM associated with Arnt deletion. Furthermore, our studies focused on CM rather than the cardiac hypertrophy studied by Krishnan et al. (36). 
A

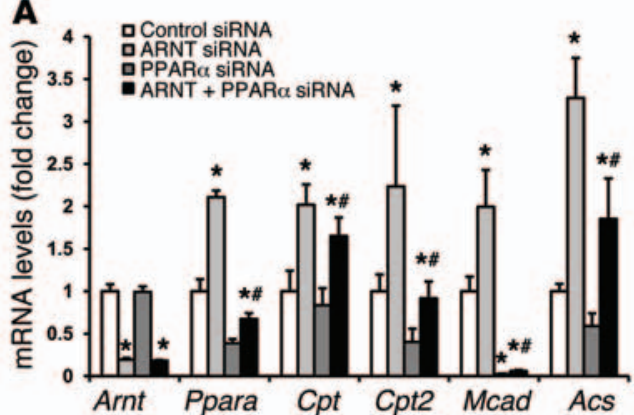

B

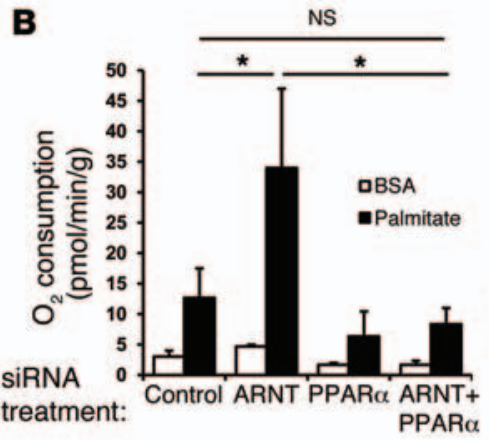

C
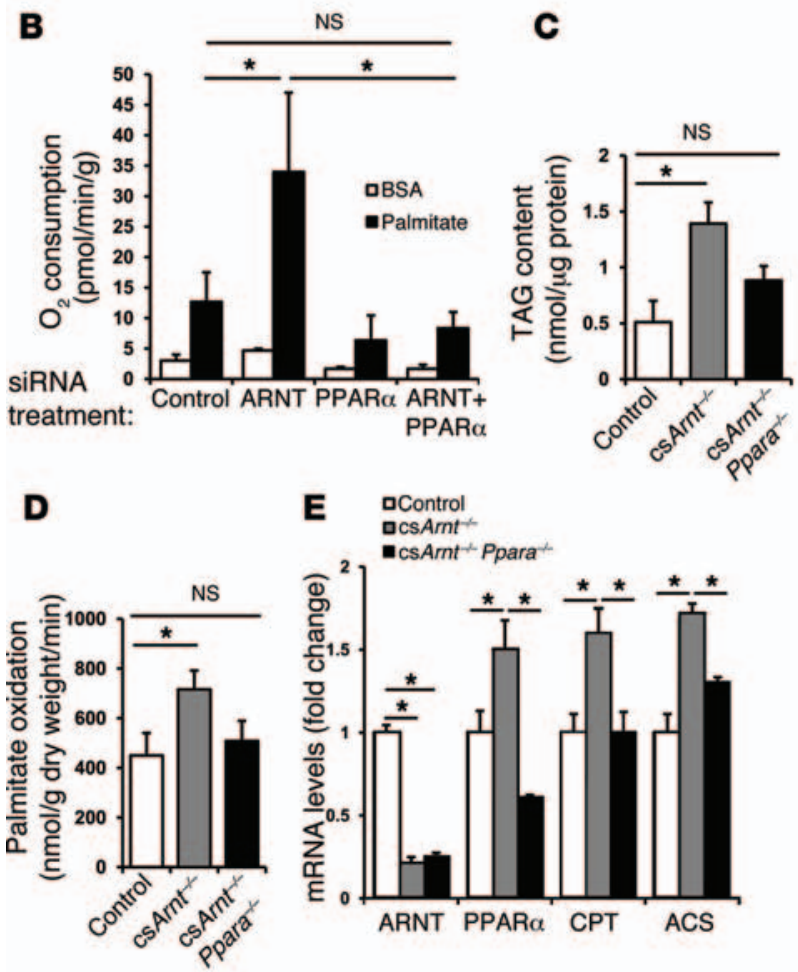

$\mathbf{F}$
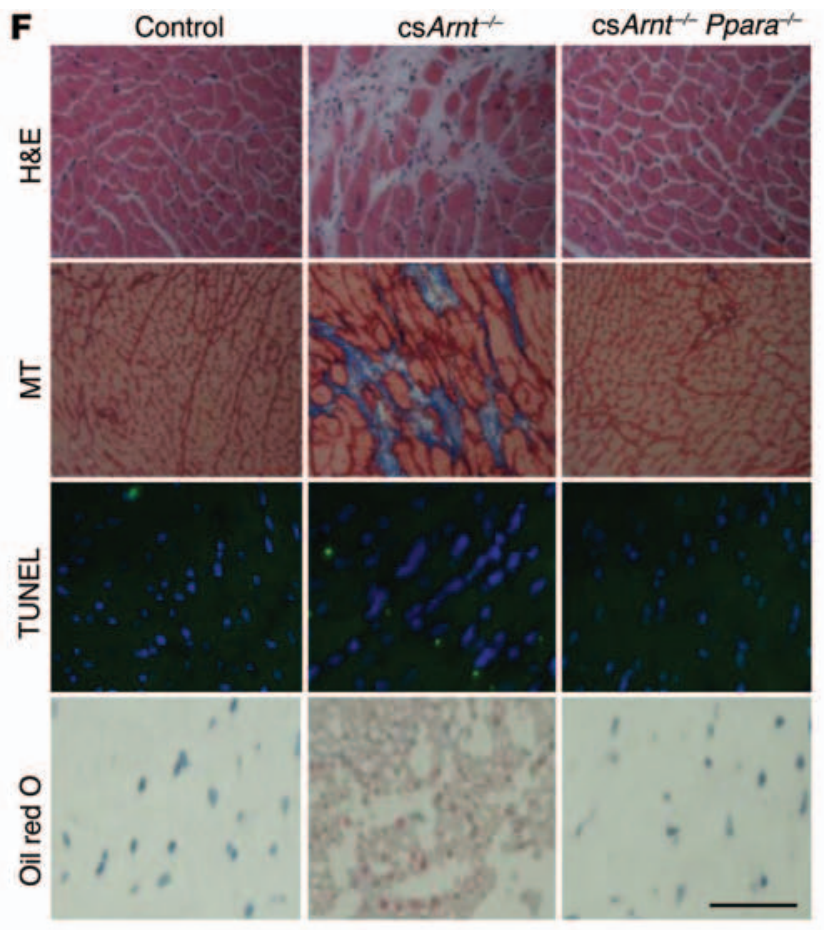

G

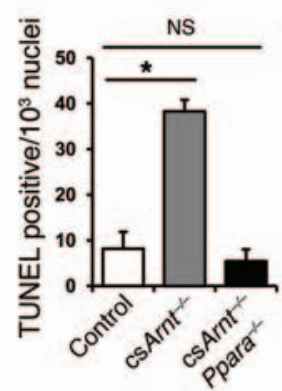

Figure 6. PPAR $\alpha$ knockdown reverses the metabolic changes associated with Arnt deletion. (A) mRNA levels of genes involved in FAU and FAO in NRCMs treated with control, ARNT, PPAR $\alpha$, and ARNT plus PPAR $\alpha$ siRNA. MCAD, medium-chain acyl-CoA dehydrogenase ( $n=6$ independent experiments). (B) FAO as determined by oxygen consumption with the exogenous addition of palmitate in NRCMs treated with control, ARNT, PPAR $\alpha$, and ARNT plus PPAR $\alpha$ siRNA ( $n=6$ independent experiments). (C) Myocardial TAG levels in control, csArnt ${ }^{-1-}$, and cs Arnt ${ }^{-1-}$ Ppara ${ }^{-/}$mice ( $n=6-8$ independent experiments). (D) Myocardial FAO determined in isolated working hearts of $\operatorname{csArnt}{ }^{-1-}, \operatorname{cs} A r n t^{-1-} P_{p a r a}^{-1-}$, or littermate controls using ${ }^{3} \mathrm{H}$-labeled palmitate $(n=5$ hearts). (E) mRNA levels of gene targets of Ppara in control, csArnt ${ }^{-1-}$, and csArnt ${ }^{-/-}$Ppara ${ }^{-1-}$ hearts $(n=6$ independent experiments). (F) Oil red O, TUNEL, MT, and H\&E staining of hearts from control, csArnt $t^{-1-}$, and ${\mathrm{cs} A r n t^{-1-}} \mathrm{Ppara}^{-/-}$mice. Scale bar: $100 \mu \mathrm{m}$. Experiments were repeated in triplicate. (C) Summary of TUNEL ${ }^{+}$ cells normalized to the number of nuclei in hearts from control, csArnt ${ }^{-1-}$, and csArnt ${ }^{-1-}$ Ppara ${ }^{-1-}$ mice $(n=4$ independent experiments). Data are presented as the mean $\pm \mathrm{SEM}$. ${ }^{*} P<0.01 ;{ }^{\#} P<0.01$ for PPAR $\alpha$ siRNA vs. ARNT plus PPAR $\alpha$ siRNA.

Nevertheless, these 2 studies provide evidence for a link between the HIF pathway and FA metabolism in the heart in response to stress.

In addition to providing substrates for oxidative phosphorylation and ATP generation, FA and TAG stores serve as important signaling molecules in the cell. Importantly, free FAs are known to bind to nuclear receptors such as PPAR $\alpha$ and stimulate their translocation into the nucleus. It is conceivable that the activation of PPAR $\alpha$ signaling we observed with Arnt deletion was secondary to the binding of free FAs to PPAR $\alpha$ and its subsequent activation. However, this mechanism is unlikely to explain all of our findings, since our luciferase studies of the Ppara promoter showed regulation of this receptor at the transcriptional level. Nevertheless, higher TAG levels in Arnt-knockout mice may further amplify PPAR $\alpha$ signaling.
In summary, our results demonstrate that deletion of Arnt in the heart leads to CM, lipid accumulation, and PPAR $\alpha$ activation. The mechanism for PPAR $\alpha$ activation occurs at the transcriptional level and through the regulation of its promoter by ARNT/ HIF $2 \alpha$. These results demonstrate a functional link between the HIF/ARNT pathway and lipid metabolism in the heart through PPAR $\alpha$ activation and provide a novel potential target for the treatment of cardiac lipotoxicity.

\section{Methods}

Animal models. csArnt ${ }^{-/-}$mice were generated by crossing Arnt $t^{t / / t}$ mice with Myh6-MCM (tamoxifen-inducible heart-specific Cre) Tg mice (purchased form The Jackson Laboratory), followed by oral adminis- 

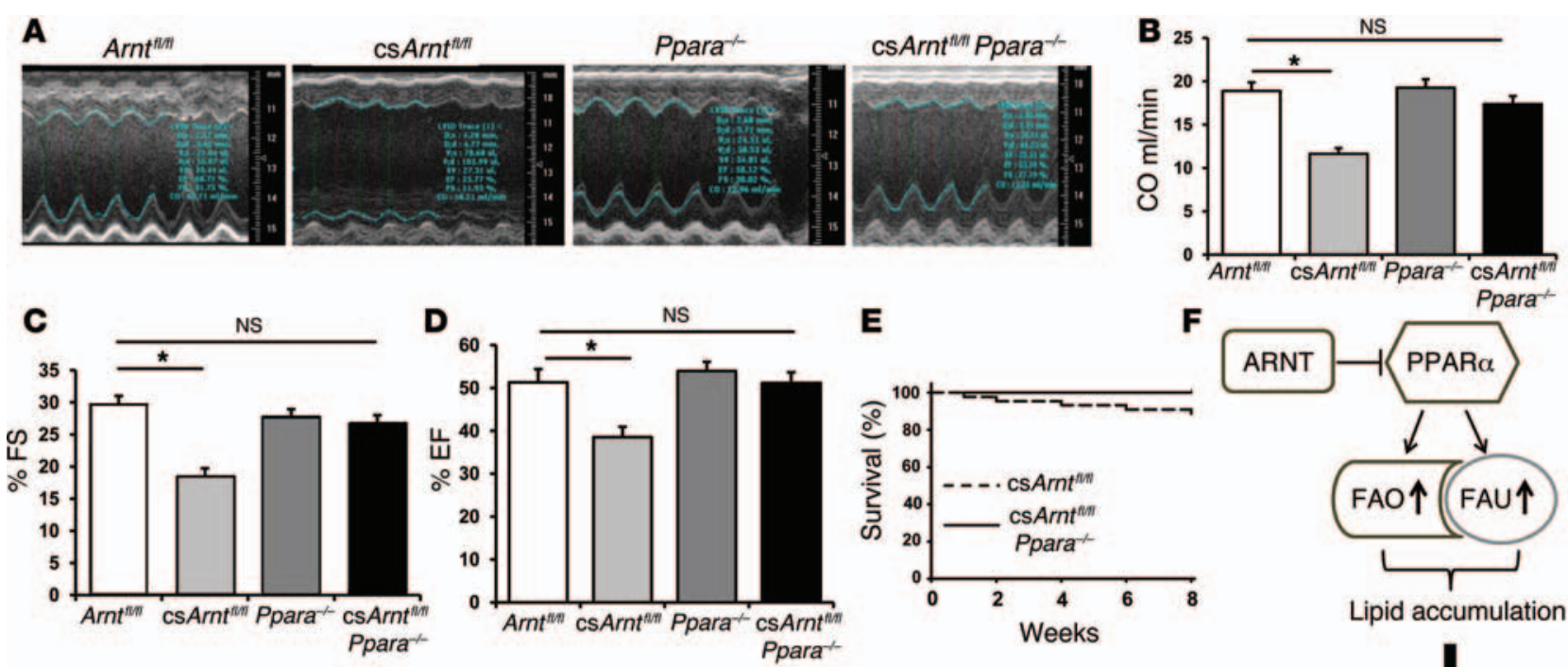

Figure 7. Ppara deletion reverses the cardiac dysfunction of csArnt $^{-/-}$mice. (A) Representative m-mode echocardiographic images of hearts from control, csArnt ${ }^{-1-}, \mathrm{Ppara}^{-/-}$, and csArnt ${ }^{-1-} \mathrm{Ppara}^{-1-}$ double-knockout mice 4 weeks after tamoxifen treatment. The experiment was repeated 7-11 times. (B-D) Echocardiographic data, including cardiac output (CO) (B), FS (C), and EF (D) from control, csArnt ${ }^{-/}$, Ppara $^{-1-}$, and $\mathrm{csArnt}^{-/-}$Ppara $^{-/-}$double-knockout mice ( $n=7-11$ independent experiments). (E) Kaplan-Meier survival curve of $\mathrm{csArnt}^{-1-}(n=35)$ and csArnt ${ }^{-1-}$ Ppara ${ }^{-/-}$double-knockout mice $(n=44)$ mice. There is a significant difference between the 2 curves based on log-rank analysis. (F) Schematic model for ARNT regulation of cardiac FA metabolism. Data are presented as the mean \pm SEM. ${ }^{*} P<0.01$.

tration of tamoxifen $(30 \mathrm{mg} / \mathrm{kg})$ for 2 weeks as described previously (37). Three sets of sex-matched littermate mice (Myh6-MCM with tamoxifen; Arnt $t^{A / f l}$ with tamoxifen; and Myh6-MCM Arnt $t^{f / / l}$ without tamoxifen) were used as controls. $\mathrm{db} / \mathrm{db}$ mice were purchased from The Jackson Laboratory and studied at 32 weeks of age. All groups of mice included a mix of males and females.

Genotyping. Genomic DNA was prepared from tail clips using the PureGene DNA isolation kit (Gentra Systems) according to the manufacturer's protocol. Approximately $10 \mathrm{ng}$ of the genomic DNA was used for PCR. Genotyping of Myh6-MCM mice was performed using 2 sets of primers. The first primer set was designed to amplify the Cre Tg construct (forward, 5'-ATACCGGAGATCATGCAA GC-3'; reverse, 5'-AGGTGGACCTGATCATGGAG-3'). The second primer set was used to amplify a mouse WT internal positive control (forward, 5'-CTAGGCCACAGAATTGAAAGATCT-3'; reverse, 5'-GTAGGTGGAAATTCTAGCATCATCC-3'). Genotyping of Arnt $t^{f / / t}$ mice was performed by PCR amplification (forward, 5 '-CACCTGAGCTAAATTACCAGGCC-3'; reverse, 5'-GCATGCTGGCACATGCCTGTCT-3'). The 2 sets of primers used for genotyping of $\mathrm{Para}^{-1}$ mice were: forward, 5'-GAGAAGTTGCAGGAGGGGATTGTG-3' (served as common); reverse: 5'-CCCATTTCGGTAGCAGGTAGTCTT-3' (WT); and reverse, 5'-GCAATCCATCTTGTTCAATGGC-3' (mutant).

Primary cell culture. NRCMs were isolated from 1- to 2-day-old Sprague-Dawley rats as previously described (38). Cardiomyocytes were cultured in DMEM/M199 (3:1), supplemented with 5\% FBS, 1.5 $\mathrm{mM}$ vitamin B12, and $1 \mathrm{mM}$ penicillin-streptomycin (Invitrogen). To prevent the proliferation of nonmyocytes, $100 \mu \mathrm{M}$ BrdU was added to the culture media (Sigma-Aldrich).

Echocardiography. Cardiac function was noninvasively monitored by transthoracic echocardiography using a VisualSonics Vevo 770 high-resolution imaging system with a $30 \mathrm{MHz}$ scanhead 1, 2, 3, and 4 weeks after oral administration of tamoxifen or normal chow. Parasternal short- and long-axis views were used to obtain 2D and M-mode images. At least 10 independent cardiac cycles per experiment were obtained.

Cardiac hemodynamic measurement. Hemodynamic studies were performed 2 weeks after tamoxifen treatment was completed, as described previously (39). A high-fidelity transducer-tipped pressure-volume catheter (Scisense Inc.) was introduced from the right carotid artery into the left ventricle of the anesthetized mouse to determine hemodynamics. Signals were digitized using a data translation series analog-digital converter and then stored and analyzed.

Histological analysis. Hearts were fixed in 10\% formalin (PBS buffered), dehydrated, and embedded in paraffin. Heart architecture was determined using transverse $5-\mu \mathrm{m}$ deparaffinized sections stained with H\&E. Fibrosis was detected with Masson trichrome staining. TUNEL positivity in the hearts from csArnt ${ }^{-/}$and control mice was determined using an in situ detection kit according to the manufacturer's instructions (Roche Diagnostics). Oil red O staining was performed on sections of unfixed, freshly frozen heart samples (6- $\mu \mathrm{m}$ in thickness).

Electron microscopy. Cardiac tissue from the left ventricle free wall was fixed with $2 \%$ glutaraldehyde in sodium cacodylate buffer at $4^{\circ} \mathrm{C}$. Fixed tissues were embedded in epoxy resin, processed for thin (70-nm) cutting with a Leica UC6 ultramicrotome, and examined with a FEI Tecnai Spirit transmission electron microscope.

Heart TAG content. Heart TAG was extracted as previously reported (40) and quantified using a Triglyceride Quantification Kit (Abcam) following the manufacturer's instructions. To measure TAG levels in isolated cells, NRCMs were homogenized in $5 \% \mathrm{NP}-40$ solution. Samples were solubilized by slowly heating to $80^{\circ} \mathrm{C}$ to $100^{\circ} \mathrm{C}$ for 5 minutes and then cooling down to room temperature. They were 
centrifuged to remove insoluble material and then diluted 10-fold in $\mathrm{ddH}_{2} \mathrm{O}$. TAG levels were determined using the Triglyceride Quantification Kit from BioVision. All samples and standards were run in triplicate, and values were normalized to protein content.

Measurement of cellular glycolysis (ECAR) and FAO. Cellular glycolysis was determined by measuring the ECAR in NRCMs using a Seahorse Bioscience XF24 extracellular flux analyzer (Billerica) as described previously (41). An increased oxygen consumption rate (OCR) immediately following the addition of palmitate substrate to the cells was used as an indicator for FAO in the XF24 Analyzer. Sixty thousand cells were seeded per well, and 24 hours later were treated for 2 subsequent days with siRNA. Forty-eight hours later, cells were used for oxygen consumption studies using unbuffered DMEM plus $1 \mathrm{mM}$ sodium pyruvate plus $1 \mathrm{~g} / \mathrm{l}$ glucose. Oxygen consumption readings were normalized to total protein per well.

2-Deoxyglucose uptake. Neonatal cardiomyocytes were grown in 6-well plates. Following serum depletion, cells were rinsed with HEPES buffer and incubated with $1 \mu \mathrm{C} \mathrm{Ci}{ }^{3} \mathrm{H}$-2-deoxy-glucose and 5 $\mathrm{mM}$ glucose per well for 10 minutes. Cells were then rinsed with icecold PBS and lysed in a buffer containing 0.1\% SDS. 2-Deoxyglucose uptake was measured for 30 seconds. Nonspecific uptake was determined in the presence of $10 \mu \mathrm{M}$ cytochalasin B, and this value was subtracted from all values. Cell-associated radioactivity was determined by lysing the cells with $0.05 \mathrm{M} \mathrm{NaOH}$, followed by measuring radioactivity using a liquid scintillation counter. Total cellular protein was determined by the Bradford method.

$F A U$. BSA-conjugated palmitate was freshly prepared with Krebs-Henseleit buffer at a final concentration of $0.0425 \mathrm{mM}$ for BSA and $0.25 \mathrm{mM}$ for palmitate. Cells were plated in a 6-well plate, rinsed with PBS buffer with $1 \%$ BSA, and incubated with $2 \mathrm{ml}$ BSA-palmitate in each well for 2 minutes at $37^{\circ} \mathrm{C}$. After washing 5 times with ice-cold PBS, cells were lysed with RIPA buffer, followed by liquid scintillation counting. Total cellular protein was determined by the Bradford method and used for normalization.

RNA isolation and quantitative real-time PCR. Total RNA from cultured NRCMs was isolated using the RNA Stat-60 reagent (TelTest Inc.), and RNA from mouse heart was isolated using an RNeasy Fibrous Tissue Mini Kit from QIAGEN according to the manufacturer's instructions. Samples of total RNA (500 ng) were reverse transcribed using the TaqMan reverse transcription reagents PCR Kit (Applied Biosystems), and the resulting cDNA was used as a PCR template. The mRNA levels of Arnt, Ppara, Pparg, Pparb, Cd36, Acs, Cpt1, Mcad, Hk2, Glut1, and Glut4 were determined by quantitative real-time PCR (qRT-PCR) with SYBR Green using the 7500 Fast RealTime PCR system (Applied Biosystems) according to the manufacturer's instructions. 18S RNA was amplified as an internal control. Relative gene expression levels were calculated using the comparative $\mathrm{Ct}$ method formula $2^{-\Delta \Delta \mathrm{Ct}}$.

siRNA treatment of NRCMs. siRNA was transfected into NRCMs using a TransMessenger Transfection Kit (QIAGEN). Forty-eight hours later, cells were lysed and used for Western blot analysis or for RNA isolation by qRT-PCR.

Western blot analysis. For Western blotting of total protein, hearts were homogenized in a modified RIPA buffer containing protease and phosphatase inhibitors (Roche). Total protein lysates were isolated from NRCMs in RIPA buffer containing protease and phosphatase inhibitors. Antibodies against ARNT, PPAR $\alpha$, HDAC1, HDAC2,
HDAC3, and HDAC4 were purchased from Cell Signaling Technology. GAPDH and tubulin antibodies were purchased from Santa Cruz Biotechnology Inc. Samples were run on an SDS-PAGE gel and transferred to a nitrocellulose membrane (Invitrogen). The protein bands were developed with an enhanced chemiluminescence substrate kit. Quantification of blots was performed using ImageJ software (NIH). (Full, uncut gels are shown in the Supplemental Material.)

Heart isolation and perfusion conditions. Hearts from $\mathrm{csArnt}^{-/-}$and littermate control mice were perfused in the working mode. Mice were anesthetized with an intraperitoneal injection of Avertin (400 $\mathrm{mg} / \mathrm{kg}$ body weight), and the hearts were subsequently excised and immersed in ice-cold Krebs-Henseleit bicarbonate solution $(118 \mathrm{mM}$ $\mathrm{NaCl}, 25 \mathrm{mM} \mathrm{NaHCO}$, $5.9 \mathrm{mM} \mathrm{KCl}, 5 \mathrm{mM}$ EDTA [pH = 7.4], $1.2 \mathrm{mM}$ $\mathrm{MgSO}_{4} \cdot 7 \mathrm{H}_{2} \mathrm{O}, 2.5 \mathrm{mM} \mathrm{CaCl} \cdot 2 \mathrm{H}_{2} \mathrm{O}$, and $5 \mathrm{mM}$ glucose). The aorta was cannulated and perfused with Krebs-Henseleit solution $\left(37^{\circ} \mathrm{C}\right)$ initiated at a hydrostatic pressure of $60 \mathrm{mmHg}$. The hearts were trimmed of excess tissue, and the opening to the left atrium was cannulated. After equilibration in the Langendorff mode, the hearts were switched to the working mode by clamping off the aortic inflow line from the Langendorff reservoir and opening the left atrial inflow line. Oxygenated Krebs-Henseleit solution consisting of $0.4 \mathrm{mM}$ palmitate bound to $3 \% \mathrm{FA}$-free BSA, $5 \mathrm{mM}$ glucose, and $1 \mathrm{mM}$ lactate in the presence of $100 \mathrm{mU} / \mathrm{ml}$ insulin was delivered to the left atrium at a preload pressure of $11.5 \mathrm{mmHg}$. Perfusate was ejected from spontaneously beating hearts into a compliance chamber and into the aortic outflow line against a hydrostatic afterload pressure of $50 \mathrm{mmHg}$. The perfusate was recirculated, and the $\mathrm{pH}$ was adjusted to 7.4 by gassing the perfusate in a glass oxygenator with a gas mixture containing $95 \% \mathrm{O}_{2}$ and $5 \%$ $\mathrm{CO}_{2}$. At the end of perfusion, the hearts were immediately frozen in liquid $\mathrm{N}_{2}$ with Wollenberger tongs and stored at $-80^{\circ} \mathrm{C}$.

Determination of glucose and palmitate oxidation. Glucose oxidation was measured by perfusing hearts with $\left[\mathrm{U}-{ }^{14} \mathrm{C}\right]$ glucose and unlabeled lactate and palmitate $(42,43)$. Palmitate oxidation measurements were performed by perfusing the hearts with glucose and $\left[9,10-{ }^{3} \mathrm{H}\right]$ palmitate. The total myocardial ${ }^{3} \mathrm{H}_{2} \mathrm{O}$ production and ${ }^{14} \mathrm{CO}_{2}$ production were determined at 10-minute intervals during the 40 - or 60-minute aerobic perfusion period. To measure the rates of palmitate oxidation, ${ }^{3} \mathrm{H}_{2} \mathrm{O}$ in perfusate samples was separated from $\left[{ }^{3} \mathrm{H}\right]$ palmitate hearts using a vapor transfer method $(42,43)$. This method consisted of adding $500 \mu \mathrm{l}$ of water into a $5-\mathrm{ml}$ scintillation vial, then placing a lidless $1.5-\mathrm{ml}$ microcentrifuge tube inside the scintillation vial. A 200- $\mu$ l perfusate sample was then added to the microcentrifuge tube, and the scintillation vial was capped. Scintillation vials were then stored initially at $50^{\circ} \mathrm{C}$ for 24 hours and then at $4^{\circ} \mathrm{C}$ for 24 hours. Following storage, the microcentrifuge tube was removed, scintillation fluid (EcoLite; Fisher Scientific) was added, and the radioactivity was counted in a liquid scintillation counter. The glucose oxidation rate was determined by quantitative measurement of ${ }^{14} \mathrm{CO}_{2}$ production from ${ }^{14} \mathrm{C}$-glucose-perfused hearts released as a gas in the oxygenation chamber and ${ }^{14} \mathrm{CO}_{2}$ dissolved as $\mathrm{H}^{14} \mathrm{CO}_{3}^{-}$in perfusate. The gaseous ${ }^{14} \mathrm{CO}_{2}$, which exits the perfusion system via an exhaust line, was trapped in hyamine hydroxide solution. The dissolved ${ }^{14} \mathrm{CO}_{2}$ as $\mathrm{H}^{14} \mathrm{CO}_{3}^{-}$was released and trapped on filter paper saturated with hyamine hydroxide in the central well of $25-\mathrm{ml}$ stoppered flasks after perfusate samples were acidified by the addition of $1 \mathrm{ml} 9 \mathrm{~N} \mathrm{H}_{2} \mathrm{SO}_{4}$.

Luciferase studies. H9EK293 cells were cotransfected using Lipofectamine Plus Reagent (Invitrogen) in a serum-free medium with both 
the recombinant firefly luciferase plasmids containing a human PPAR $\alpha$ promoter-luciferase reporter (200 ng) and the Renilla luciferase plasmid ( $25 \mathrm{ng}$ ), along with $25 \mathrm{nM}$ of either ARNT siRNA or control siRNA. Forty-eight hours after transfection, cells were collected, and the luciferase signal intensity was measured with a dual-luciferase reporter assay kit (Promega) on a Berthold Lumat LB 9570 luminometer. Firefly luciferase expression was corrected by Renilla luciferase expression in the same well to normalize for variations in transfection efficiency.

Mutagenesis of the HRE site was performed using PCR-based sitedirected mutagenesis. Briefly, primers containing the desired mutations were used to amplify the Ppara promoter-luciferase reporter plasmid using the HiFi PCR Kit (Kapa Biosystems), followed by DpnI (New England Biolabs Inc.) digestion to remove the original template. The following primers were used for the HRE sequence, with the mutation indicated in bold letters: sense primer,GGGCCGAGGGGCGGTTATGCTCGCGGGGGCGCGGC; anti-sense primer, GCCGCGCCCCCGCGAGCATAACCGCCCCTCGGCCC. The sequence of the resulting plasmid was verified before subsequent experiments.

ChIP studies. Confluent HepG2 culture was harvested with trypsin and cross-linked with $1.1 \%$ formaldehyde. ChIP was performed using anti-ARNT antibody (Cell Signaling Technology) as described previously (44). After reverse cross-linking and proteinase K and RNAse A digestion, DNA was purified with a QIAGEN PCR purification kit. Purified DNA was used as input for qRT-PCR. For sequential ChIP, chromatin from the first IP was eluted with elution buffer containing $5 \mathrm{mM}$ DTT and diluted 10-fold before being used as input for the second IP. The second IP was performed as described previously (44).
The positive control genes were identified in earlier studies, and the primer sequences for those genes have been validated as described previously (45). For ChIP against acetylated histones, sodium butyrate was added to all buffers. Anti-HIF $2 \alpha$ antibody was purchased from Novus, and acetylated histone $\mathrm{H} 3$ and acetylated histone $\mathrm{H} 4$ antibodies were purchased from Millipore.

Statistics. Data are expressed as the mean \pm SEM. Statistical significance was assessed with ANOVA. For multiple group comparisons, a post-hoc Tukey's test was performed when ANOVA reached significance. A $P$ value of less than 0.05 was considered statistically significant.

Study approval. All animal studies were reviewed and approved by the IACUC of Northwestern University.

\section{Acknowledgments}

We would like to thank Konrad Sawicki for careful review of the manuscript. Imaging work was performed at the Northwestern University Cell Imaging Facility, which is supported by NCI grant CCSG P30 CA060553, awarded to the Robert H. Lurie Comprehensive Cancer Center. H. Ardehali is supported by NIH grants K02 HL107448, R01 HL104181, and 1PO1 HL108795. $\mathrm{R}$. $\mathrm{Wu}$ is supported by American Heart Association (AHA) grant 13SDG17270046.

Address correspondence to: Hossein Ardehali, Tarry 14-733, 303 E. Chicago Ave., Chicago, Illinois 60611, USA. Phone: 312.503.2342; E-mail: h-ardehali@northwestern.edu.
1. Wild S, Roglic G, Green A, Sicree R, King H. Global prevalence of diabetes: estimates for the year 2000 and projections for 2030. Diabetes Care. 2004;27(5):1047-1053.

2. Battiprolu PK, Lopez-Crisosto C, Wang ZV, Nemchenko A, Lavandero S, Hill JA. Diabetic cardiomyopathy and metabolic remodeling of the heart. Life Sci. 2013;92(11):609-615.

3. Dirkx E, Schwenk RW, Glatz JF, Luiken JJ, van Eys GJ. High fat diet induced diabetic cardiomyopathy. Prostaglandins Leukot Essent Fatty Acids. 2011;85(5):219-225.

4. van de Weijer T, Schrauwen-Hinderling VB, Schrauwen P. Lipotoxicity in type 2 diabetic cardiomyopathy. Cardiovasc Res. 2011;92(1):10-18.

5. Ema M, et al. Two new members of the murine Sim gene family are transcriptional repressors and show different expression patterns during mouse embryogenesis. Mol Cell Biol. 1996;16(10):5865-5875.

6. Rowlands JC, Gustafsson JA. Aryl hydrocarbon receptor-mediated signal transduction. Crit Rev Toxicol. 1997;27(2):109-134.

7. Semenza GL. HIF-1: mediator of physiological and pathophysiological responses to hypoxia. JAppl Physiol. 2000;88(4):1474-1480.

8. Bugger H, Abel ED. Molecular mechanisms for myocardial mitochondrial dysfunction in the metabolic syndrome. Clin Sci (Lond). 2008;114(3):195-210.

9. Maltepe E, Schmidt JV, Baunoch D, Bradfield CA, Simon MC. Abnormal angiogenesis and responses to glucose and oxygen deprivation in mice lacking the protein ARNT. Nature.
1997;386(6623):403-407.

10. Gunton JE, et al. Loss of ARNT/HIF1 $\beta$ mediates altered gene expression and pancreatic-islet dysfunction in human type 2 diabetes. Cell. 2005;122(3):337-349.

11. Wang XL, et al. Ablation of ARNT/HIF1 $\beta$ in liver alters gluconeogenesis, lipogenic gene expression, and serum ketones. Cell Metab. 2009;9(5):428-439.

12. Robinson E, Grieve DJ. Significance of peroxisome proliferator-activated receptors in the cardiovascular system in health and disease. Pharmacol Ther. 2009;122(3):246-263.

13. Fruchart JC. Peroxisome proliferator-activated receptor-alpha $(\operatorname{PPAR} \alpha)$ : at the crossroads of obesity, diabetes and cardiovascular disease. Atherosclerosis. 2009;205(1):1-8.

14. Madrazo JA, Kelly DP. The PPAR trio: regulators of myocardial energy metabolism in health and disease. JMol Cell Cardiol. 2008;44(6):968-975.

15. Bortolini M, Wright MB, Bopst M, Balas B. Examining the safety of PPAR agonists - current trends and future prospects. Expert Opin Drug Saf. 2013;12(1):65-79.

16. Chinetti G, et al. Activation of proliferator-activated receptors $\alpha$ and $\gamma$ induces apoptosis of human monocyte-derived macrophages. J Biol Chem. 1998;273(40):25573-25580.

17. Chinetti $G$, et al. PPAR- $\alpha$ and PPAR- $\gamma$ activators induce cholesterol removal from human macrophage foam cells through stimulation of the ABCA1 pathway. Nat Med. 2001;7(1):53-58.

18. Delerive P, et al. Peroxisome proliferator-activated receptor alpha negatively regulates the vascular inflammatory gene response by negative crosstalk with transcription factors NF- $\mathrm{BB}$ and $\mathrm{AP}-1$. JBiol Chem. 1999;274(45):32048-32054.

19. Finck BN, et al. The cardiac phenotype induced by PPARalpha overexpression mimics that caused by diabetes mellitus. J Clin Invest. 2002;109(1):121-130.

20. Djouadi F, et al. A gender-related defect in lipid metabolism and glucose homeostasis in peroxisome proliferator- activated receptor alpha- deficient mice. J Clin Invest. 1998;102(6):1083-1091.

21. Park SY, et al. Cardiac-specific overexpression of peroxisome proliferator-activated receptor- $\alpha$ causes insulin resistance in heart and liver. Diabetes. 2005;54(9):2514-2524.

22. Finck BN, et al. A critical role for PPAR $\alpha$-mediated lipotoxicity in the pathogenesis of diabetic cardiomyopathy: modulation by dietary fat content. Proc Natl Acad Sci U S A. 2003;100(3):1226-1231.

23. Doenst T, Bugger H, Schwarzer M, Faerber G, Borger MA, Mohr FW. Three good reasons for heart surgeons to understand cardiac metabolism. Eur J Cardiothorac Surg. 2008; 33(5):862-871.

24. Sohal DS, et al. Temporally regulated and tissue-specific gene manipulations in the adult and embryonic heart using a tamoxifen-inducible Cre protein. Circ Res. 2001;89(1):20-25.

25. Koitabashi N, et al. Avoidance of transient cardiomyopathy in cardiomyocyte-targeted tamoxifen-induced MerCreMer gene deletion models. Circ Res. 2009;105(1):12-15.

26. Son NH, et al. PPAR $\gamma$-induced cardiolipotoxicity in mice is ameliorated by PPAR $\alpha$ deficiency 


\section{RESEARCH ARTICLE}

despite increases in fatty acid oxidation. JClin Invest. 2010;120(10):3443-3454.

27. Eltzschig HK, et al. HIF-1-dependent repression of equilibrative nucleoside transporter (ENT) in hypoxia. JExp Med. 2005;202(11):1493-1505.

28. Krishnan J, et al. Dietary obesity-associated Hifl $\alpha$ activation in adipocytes restricts fatty acid oxidation and energy expenditure via suppression of the Sirt2-NAD system. Genes Dev. 2012;26(3):259-270.

29. Wang Y, et al. Regulation of endocytosis via the oxygen-sensing pathway. Nat Med. 2009;15(3):319-324.

30. Kaelin WG Jr. How oxygen makes its presence felt. Genes Dev. 2002;16(12):1441-1445.

31. Iyer NV, et al. Cellular and developmental control of $\mathrm{O} 2$ homeostasis by hypoxia-inducible factor 1 a. Genes Dev. 1998;12(2):149-162.

32. Ryan HE, Lo J, Johnson RS. HIF-1 $\alpha$ is required for solid tumor formation and embryonic vascularization. ЕМВО J. 1998;17(11):3005-3015.

33. Huang Y, et al. Cardiac myocyte-specific HIF- $1 \alpha$ deletion alters vascularization, energy availabil- ity, calcium flux, and contractility in the normoxic heart. FASEB J. 2004;18(10):1138-1140.

34. Sano M, et al. p53-induced inhibition of Hif-1 causes cardiac dysfunction during pressure overload. Nature. 2007;446(7134):444-448.

35. Scortegagna $\mathrm{M}$, et al. Multiple organ pathology, metabolic abnormalities and impaired homeostasis of reactive oxygen species in Epas1 $1^{-/}$mice. Nat Genet. 2003;35(4):331-340.

36. Krishnan J, et al. Activation of a HIF1 $\alpha-P P A R \gamma$ axis underlies the integration of glycolytic and lipid anabolic pathways in pathologic cardiac hypertrophy. Cell Metab. 2009;9(6):512-524.

37. Ichikawa Y, et al. Disruption of ATP-binding cassette B8 in mice leads to cardiomyopathy through a decrease in mitochondrial iron export. Proc Natl Acad Sci U S A. 2012;109(11):4152-4157.

38. Ardehali H, O'Rourke B, Marban E. Cardioprotective role of the mitochondrial ATP-binding cassette protein 1. Circ Res. 2005;97(8):740-742.

39. Wu R, et al. Reduction in hexokinase II levels results in decreased cardiac function and altered remodeling after ischemia/reperfusion injury.
Circ Res. 2011;108(1):60-69.

40. Marsili A, et al. Mice with a targeted deletion of the type 2 deiodinase are insulin resistant and susceptible to diet induced obesity. PLoS One. 2011;6(6):e20832.

41. Guo $S$, et al. A cell-based phenotypic assay to identify cardioprotective agents. Circ Res. 2012;110(7):948-957.

42. Barr RL, Lopaschuk GD. Direct measurement of energy metabolism in the isolated working rat heart. J Pharmacol Toxicol Methods. 1997;38(1):11-17.

43. Lopaschuk GD, Barr RL. Measurements of fatty acid and carbohydrate metabolism in the isolated working rat heart. Mol Cell Biochem. 1997;172(1-2):137-147.

44. Huang Z, et al. GATA-2 reinforces megakaryocyte development in the absence of GATA-1. Mol Cell Biol. 2009;29(18):5168-5180.

45. Schodel J, Oikonomopoulos S, Ragoussis J, Pugh CW, Ratcliffe PJ, Mole DR. High-resolution genome-wide mapping of HIF-binding sites by ChIP-seq. Blood. 2011;117(23):e207-e217. 